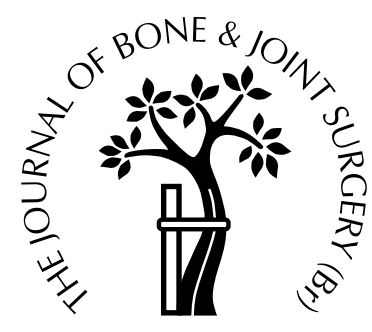

\title{
Histological evaluation of chondral defects after autologous chondrocyte implantation of the knee
}

\author{
T. W. R. Briggs, S. Mahroof, L. A. David, J. Flannelly, J. Pringle, M. Bayliss \\ From the Royal National Orthopaedic Hospital, Stanmore, England
}

W e have performed a prospective, single-surgeon study analysing the histological results of autologous chondrocyte implantation.

Fourteen patients underwent autologous chondrocyte implantation of the knee and were evaluated at one year by clinical assessment and arthroscopy. Standard staining was used to examine the sections. In addition, in situ hybridisation was used to establish type-IIa and type-IIb collagen mRNA expression and immunolocalisation techniques demonstrated the positions of type-II and type-X collagen.

Eight patients regenerated hyaline cartilage and also contained type-X collagen in the deepest layers and type-II collagen in the deep layers. Three demonstrated fibrocartilage and had type-II collagen in the deep layers. In situ hybridisation revealed that all 14 samples had the potential to express both type-IIa and type-IIb collagen.

We have shown that one year after the initial implantation chondrocytes are capable of producing type-II collagen and that they continue to proliferate and mature.

J Bone Joint Surg [Br] 2003;85-B:1077-83. Received 5 July 2002; Accepted after revision 14 April 2003

Fibrocartilage is formed after injury to the articular surface of the knee. Techniques used to treat chondral defects include abrasion arthroplasty, drilling and microfracture but

T. W. R. Briggs, MCh Orth, Consultant Orthopaedic Surgeon S. Mahroof, MRCS, Specialist Registrar, Orthopaedics and Trauma Surgery L. A. David, MRCS, Specialist Registrar, Orthopaedics and Trauma Surgery J. Pringle, FRCS, Consultant Histopathologist

Bone Tumour Unit, Royal National Orthopaedic Hospital Trust, Brockley Hill, Stanmore, Middlesex HA7 4LP, UK.

J. Flannelly, $\mathrm{PhD}$, Research Associate

M. Bayliss, PhD, Professor of Connective Tissue Biochemistry

Department of Veterinary Basic Sciences, Royal Veterinary College, Royal College Street, London NW1 0TU, UK.

Correspondence should be sent to Mr T. W. R. Briggs.

(C)2003 British Editorial Society of Bone and Joint Surgery doi:10.1302/0301-620X.85B7.13672 \$2.00 have had variable results. ${ }^{1-5}$ The fibrocartilage formed, however, has a lower load-bearing capacity than normal fibrocartilage and biomechanical characteristics inferior to those of hyaline cartilage which invariably gives only a short-term recovery. ${ }^{6}$ The technique of implantation of autologous chondrocytes has been developed to allow the regeneration of hyaline-like cartilage in order to delay and possibly prevent the onset of degenerative osteoarthritis. It is now an accepted form of treatment for defects of the articular cartilage of the knee..$^{7-12}$

It has been shown that the neocartilage not only resembles hyaline cartilage but also contains most components of normal hyaline cartilage. ${ }^{8,10-12}$ No studies so far have identified a similar superficial layer to normal articular hyaline cartilage, hence the term hyaline-like as opposed to hyaline has been used to describe the engineered cartilage. ${ }^{7-12}$ In fact, the superficial layer in the neocartilage is fibrocartilaginous in nature.

Type-II collagen makes up $90 \%$ of the collagenous matrix in hyaline cartilage and differentiates hyaline cartilage from fibrocartilage which contains no type-II collagen. ${ }^{13-15}$ The latter consists of two variants, type-IIa, usually present in a chondroprogenitor cellular phenotype and found in developing skeletal tissue, and type-IIb, which is expressed by mature chondrocytes. Studies have indicated that the presence of type-IIa collagen is associated with a reversion of mature chondrocytes to a chondroprogenitor phenotype in normal articular cartilage and may be a marker of the breakdown and regeneration of cartilage as seen in osteoarthritis. ${ }^{16}$ Immature chondroprogenitor cells may be essential for the regeneration observed in autologous chondrocyte implants. Detection of mRNA for type-IIa collagen by in situ hybridisation would suggest that such cells are present.

Most centres have used periosteal flaps to seal chondrocytes into the defect. ${ }^{7-12}$ It is possible that mesenchymal stem cells from the periosteal flap may also be involved in the regeneration of neocartilage in these studies. $^{9}$ An inert, absorbable type-I/III collagen membrane (Chondro-Gide; Geistlich Biomaterials, Wolhusen, Switzerland) has been developed for containing chondrocytes in the chondral defect. ${ }^{17}$ As yet, there have been no published clinical data of the use of this membrane with this technique. 


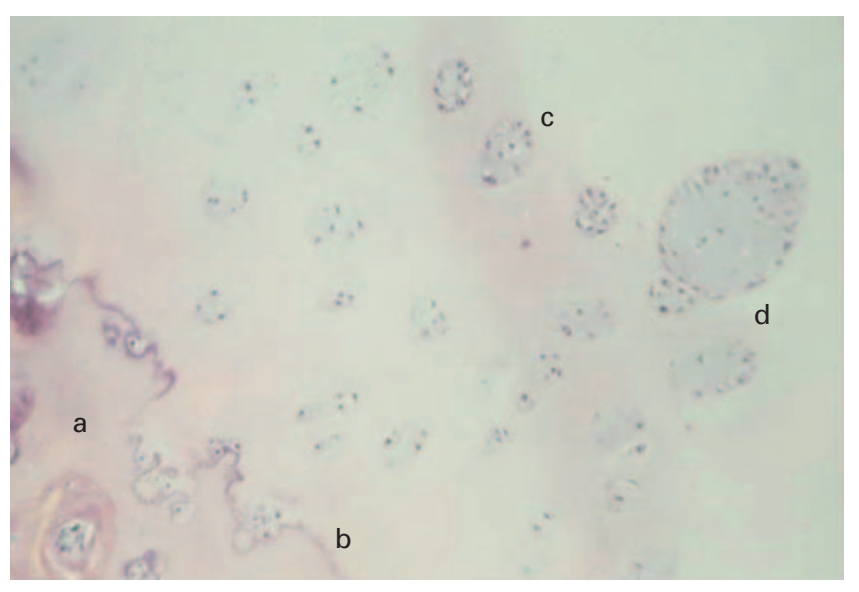

Fig. 1

Photomicrograph showing a) subchondral bone, b) the tidemark and c) and d) the deep zone of the cartilage. There is focal remodelling of the subchondral bone and areas of enchondral ossification (haematoxylin and eosin x 50).

Our aim was to assess the nature of the cartilage produced by chondrocytes in chondral defects one year after autologous chondrocyte implantation using an inert type-I/ III collagen membrane. We also examined the distribution of collagen type II and type $\mathrm{X}$ in the neocartilage.

\section{Patients and Methods}

Ethical approval was obtained and patients consented to have autologous chondrocyte implantation using a synthetic membrane and an arthroscopy with a biopsy of the treated unilateral chondral defect one year after implantation. Fourteen patients (four women and ten men) with a median age of 30 years (16 to 51) were recruited over a period of two years as part of a single-surgeon, prospective study. The anatomical site of the defect was the medial femoral condyle in eight, the lateral femoral condyle in four, the trochlea in one and the patella in one. The mean area of the defect was $2.46 \pm 2.45 \mathrm{~cm}^{2}(\mathrm{SD})$. All patients had sporting interests, physical hobbies or physical occupations. The presenting symptoms were pain (100\%), giving way (64\%), swelling (57\%), locking (36\%), stiffness (29\%) and clicking (1\%). The onset of symptoms was gradual in eight knees and acute in six. The cause of the symptoms was a sports injury (nine knees), a fall (three), chondromalacia (one) and unknown (one). Clinical examination revealed local tenderness in eight knees, an effusion in four, anterior drawer in two, muscle wasting in two and crepitus in one. One knee was normal on examination.

Twelve of the patients had received conservative treatment before surgery, including physiotherapy (six), analgesics (five), bracing (four) and immobilisation in plaster of Paris (one). All 14 patients had also undergone previous surgical procedures, including arthroscopic washout (11), multiple washout (five), arthroscopic debridement of menisci (four), reconstruction of the anterior cruciate ligament (four) and removal of a loose body (one). Seven patients had had multiple procedures.

Operative technique. At the time of transplantation the entire defect including a thin layer of subchondral bone was curetted and submitted for histological examination. The patients were all treated using cells grown by Verigen A/S (Kastrup, Denmark) according to the technique used by Peterson et al. ${ }^{7}$ Instead of using a periosteal flap, our technique involved the use of an inert type-I/III collagen membrane which was sutured to close the defect. ${ }^{17}$ This was then sealed with a fibrin glue. The patient was immobilised postoperatively in plaster of Paris for two weeks after which gentle non-weight-bearing physiotherapy was introduced for six weeks. Sporting activity was gradually increased after six months but strenuous sporting activity was withheld for up to 12 months.

Clinical assessment. Pre-operative clinical assessment included a verbal numerical pain score, the activity-scale of Tegner and Lysholm, ${ }^{18}$ the score of Lysholm and Gillquist, ${ }^{19}$ the Brittberg rating ${ }^{8}$ and a clinical examination. This assessment was repeated one year after operation with further assessments made on an annual basis. An arthroscopy was performed on all patients at about a year (mean 11 months) after surgery. At arthroscopy, two $3 \mathrm{~mm}$ core biopsies were taken from the centre of the transplanted area (Jamshidi needle, $3 \mathrm{~mm}$ diameter). The transplanted areas have a visible border viewed easily identified down the arthroscope. The cores were submitted for basic histology, in situ hybridisation and immunolocalisation.

\section{Histological assessment}

Basic histology. Initial histological assessment was performed by a consultant histopathologist (JP). The biopsy core was fixed in $10 \%$ neutral buffered formalin for 24 hours. After this, the core was decalcified in EDTA incubated at $45^{\circ} \mathrm{C}$. The endpoint of decalcification was assessed by taking radiographs of the samples in a modern Faxitron. After routine processing and cutting, the sections were stained with Erlich's haematoxylin and eosin and Safranin $\mathrm{O}$ (Figs 1 and 2). Two coated spares were prepared for routine immunohistochemistry. The remainder of the tissue was embedded in the paraffin block for ease of transport and then rehydrated and frozen before in situ hybridisation and immunolocalisation.

The sections were examined by transmitted and polarised light microscopy and by immunohistochemical analysis for S100 protein (Fig. 3). Normal human tissues which express S100 protein include chondrocytes, adipocytes, Schwann cells and other cells of neural origin. S100 was used to confirm the presence of chondrocytes in our study.

The following criteria were used to classify the biopsies as either hyaline cartilage, fibrocartilage or fibrous tissue.

Hyaline cartilage. This has a distinctive architecture. The deep zone may contain scattered clusters of chondrocytes. The major portion consists of evenly distributed chondrocytes lying in lacunae associated with evenly staining 


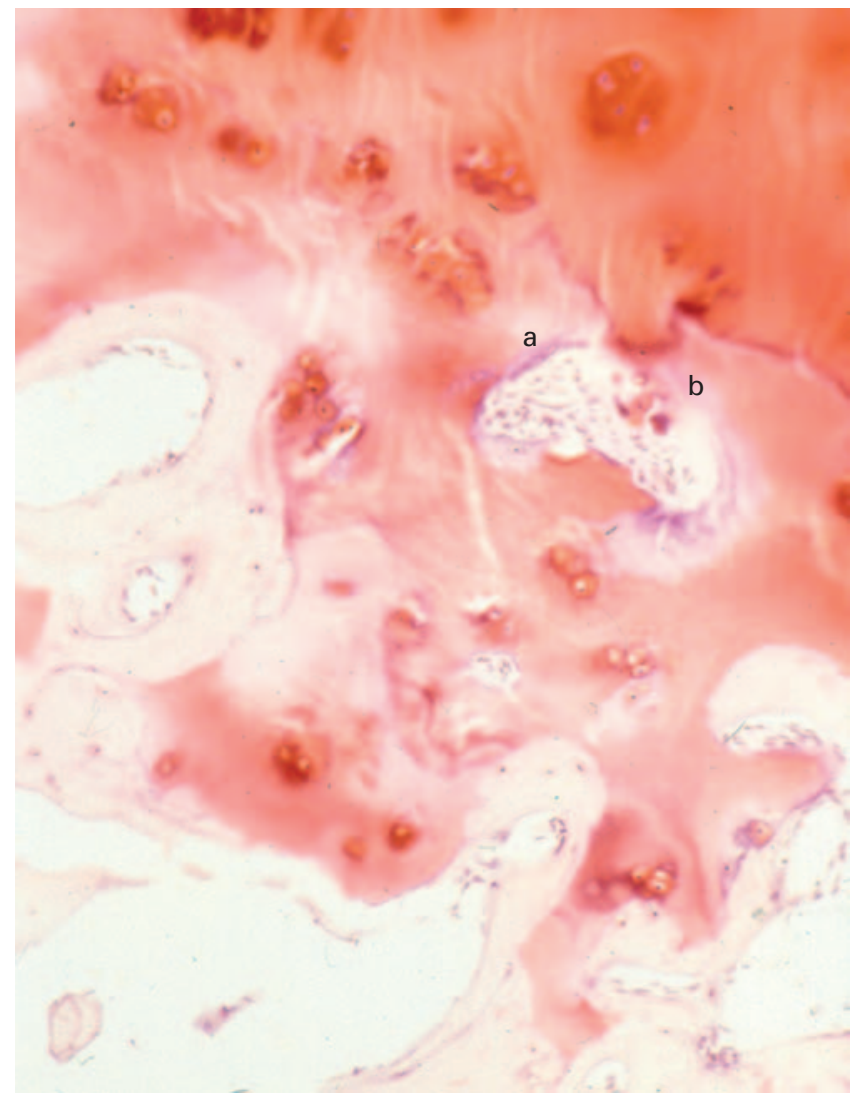

Fig. 2

Photomicrograph showing an area of hyaline cartilage similar to that in Figure 1 with a) areas of enchondral ossification and b) focal remodelling of the subchondral bone adjacent to the tidemark (Safranin O x 70).

matrix which is positive for special stains such as Safranin O. With polarised light there is no evidence of collagen fibrils giving a smooth, glassy appearance. The superficial zone contains elongated chondrocytes with their long axis parallel to the surface. The chondrocytes show strong expression of S100 protein.

Fibrocartilage. This has a rather disorganised appearance and lacks the heterogenous zonality found in hyaline cartilage. Under polarised light, a meshwork of collagen fibrils is present throughout. There is strong matrix staining with Safranin O. The chondrocytes show similar expression of S100 protein as seen in hyaline cartilage.

Fibrous tissue. This consists of fibroblasts distributed in a matrix containing variable amounts of collagen. Under polarised light, bands and fibrils of collagen are easily identified. Staining of this tissue for Safranin O and S100 is negative.

Immunolocalisation. The protocol for detecting collagen antigens using polyclonal antibodies followed that described by Chambers et al. ${ }^{20}$ Frozen tissue sections were first fixed to ten minutes by immersing them in cooled acetone, then air dried and quenched for endogenous peroxidase activity by immersion in hydrogen peroxide/methanol

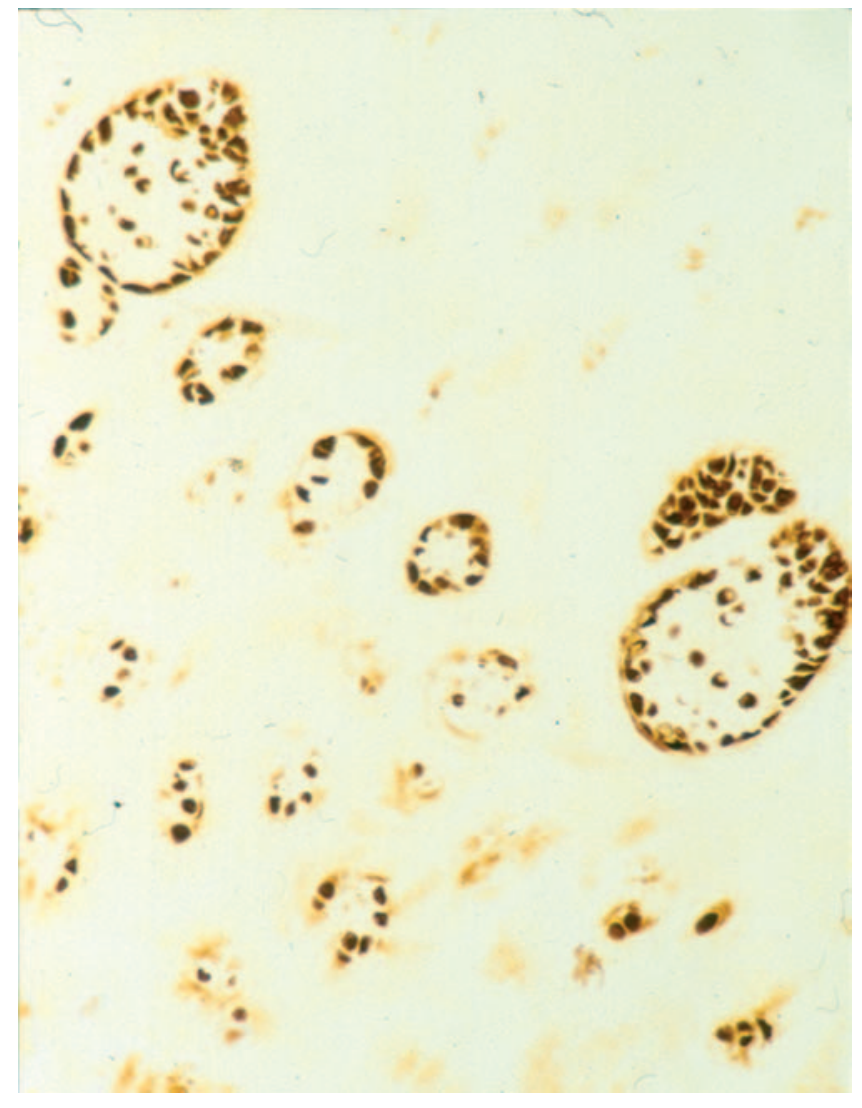

Fig. 3

Photomicrograph showing the deep zone of the hyaline cartilage with a uniform strong nuclear expression for $\mathrm{S} 100$ protein in the chondrocyte nuclei (S100 protein $\times 70)$.

solution $(0.03 \%)$ for two minutes. Sections were then digested with protease-free chondroitinase ABC lyase (ICN Biomedicals Inc, Costa Mesa, California; 0.02 units per ml, $0.1 \mathrm{M}$ Tris- $\mathrm{HCl}, \mathrm{pH}$ 8.8, 0.03M sodium acetate) for $30 \mathrm{~min}-$ utes at $37^{\circ} \mathrm{C}$ and washed in phosphate-buffered saline (PBS).

Slides were incubated with normal serum in PBS to block non-specific activity for one hour at room temperature (Vector Laboratories, Peterborough, UK). The primary antibody was incubated overnight at $4^{\circ} \mathrm{C}$ in a humidified chamber with goat anti-collagen antibodies at a concentration of $50 \mu \mathrm{g} / \mathrm{ml}-1$ in PBS containing 5\% normal serum (Santa Cruz Biotechnology Inc, Santa Cruz, California). The original immunising peptide was used at the same concentration as a negative control also supplied by Santa Cruz Biotechnology. Unbound antibody was removed by washing with PBS, and bound antibody was detected using a biotinylated swine secondary antibody for one hour at room temperature (1:2000 dilution; Dako Diagnostics Ltd, Ely, UK). The bound secondary antibody was detected with a biotinstreptavadin detection system (Vector ABC reagent; Vector Laboratories). The peroxidase was detected using a DAB detection kit (Vector Laboratories) according to the manu- 


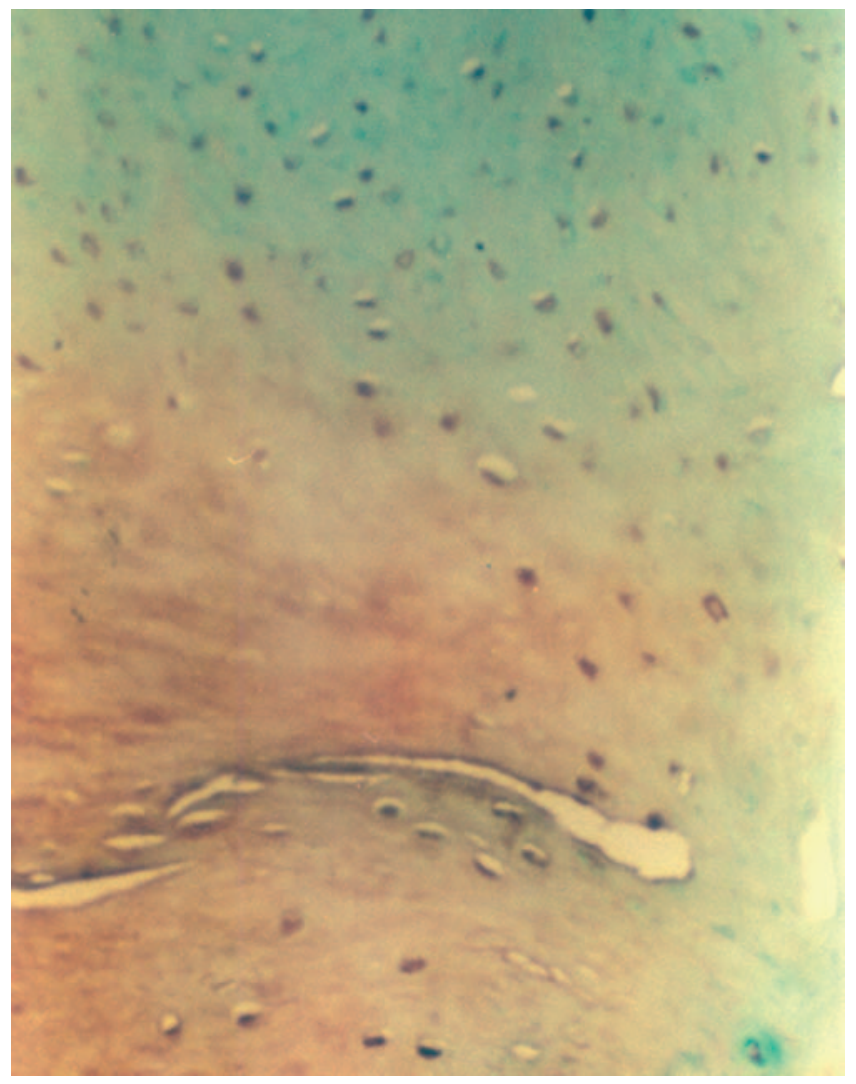

Fig. 4

Photomicrograph showing positive staining for collagen type II in the deep zone of the cartilage and negative staining in the superficial zone (polyclonal antibody to collagen type II x 70).

facturer's instructions. The sections were finally rinsed in distilled water, air dried and mounted with aquamount (VWR International Ltd, Poole, UK).

Staining for type-II and type-X collagen was recorded as its presence or absence in the cartilage and in some cases sections were counterstained with Methyl Green (Vector Laboratories) to provide contrast between the chondrocytes and the cartilage matrix. The positions of type-II and type-X collagen were also noted (Figs 4 and 5).

In situ hybridisation. The protocol for in situ hybridisation was essentially carried out using the method described by Flannelly et al. ${ }^{21}$ The probes for type-IIa and type-IIb collagen (sense and antisense oligonucleotide probes) were prepared commercially ( $\mathrm{VH}$ Bio Ltd, UK), to contain one molecule of digoxygenin-dUTP at both the 3 ' and 5 ' ends. Briefly, sections were thawed at room temperature and fixed in freshly prepared 4\% paraformaldehyde in PBS for 20 minutes, washed twice in PBS for five minutes and digested with proteinase $\mathrm{K}(20 \mu \mathrm{gml}-1)$ in $50 \mathrm{mM}$ Tris- $\mathrm{HCl}$ buffer and $5 \mathrm{mM}$ EDTA $(\mathrm{pH} 8)$ for ten minutes at $37^{\circ} \mathrm{C}$. The sections were then washed in PBS, refixed in $4 \%$ paraformaldehyde for 20 minutes and rinsed in distilled water. Before hybridisation they were treated with $0.25 \%$ acetic anhydride

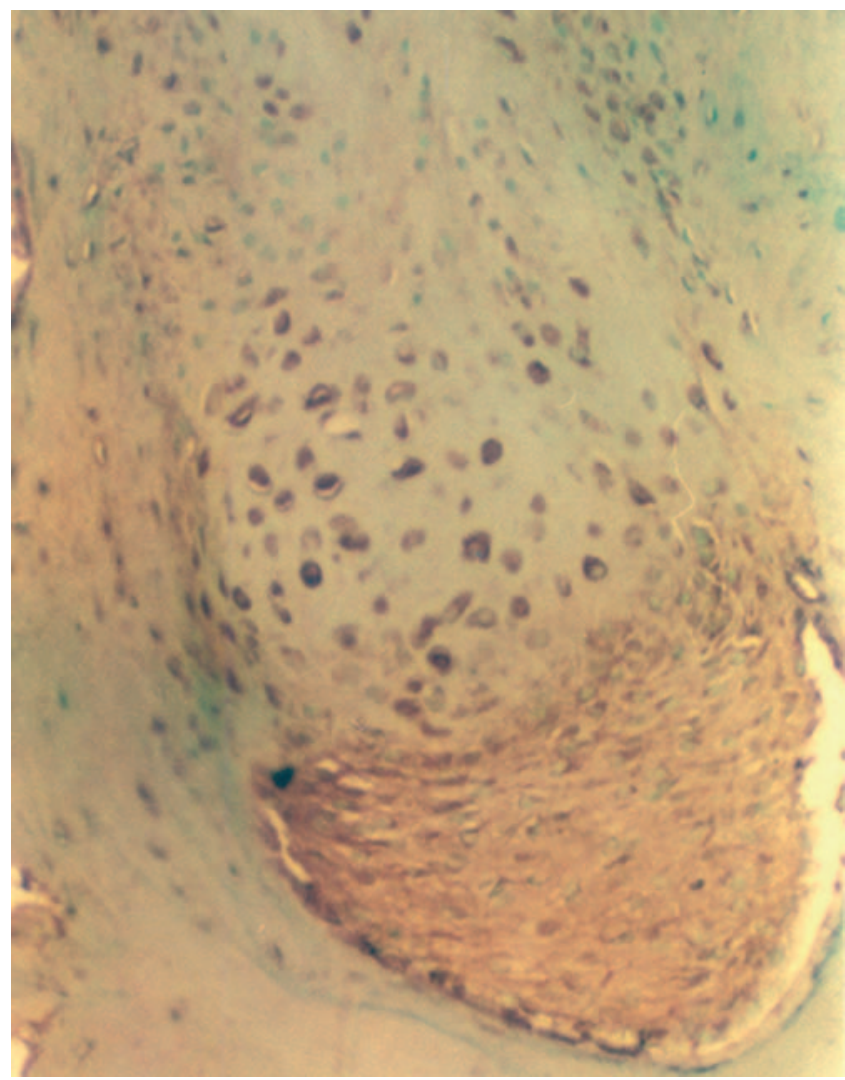

Fig. 5

Photomicrograph showing positive staining for collagen type $\mathrm{X}$ in the deepest zone of the cartilage closest to bone at the bottom of the picture (polyclonal antibody to collagen type X x 70).

in $0.1 \mathrm{M}$ triethanolamine $\mathrm{HCl}, \mathrm{pH} 8$ for ten minutes followed by two washes in PBS and then air-dried.

Oligonucleotide sense and antisense probes were used at a concentration of $2.5 \mathrm{ng} / \mathrm{ml}^{-1}$ in hybridisation buffer (Amersham International PLC, Amersham, UK). The hybridisation solution was applied to each section before hybridisation overnight at $42^{\circ} \mathrm{C}$. Control sections were exposed to hybridisation solution with no probe, or were RNAase treated before hybridisation with antisense or sense oligonucleotide probes. Sections were then washed, initially in $5 \mathrm{X}$ (standard saline citrate, SSC; $\mathrm{pH} 7.0$ ) and then in $2 \mathrm{X}$ $\mathrm{SSC}, 50 \%$ formamide (Sigma-Aldrich, Poole, UK) at $50^{\circ} \mathrm{C}$. This was followed by sequential washes in $2 \mathrm{X} \mathrm{SSC}$ and 0.1 $\mathrm{X}$ SSC for ten minutes each at room temperature. The sections were rinsed briefly in a maleic acid buffer $(0.1 \mathrm{M}$ maleic acid, $0.15 \mathrm{M}$ sodium chloride, $\mathrm{pH}$ 7.5) and then blocked with $0.5 \%$ blocking solution in maleic buffer for 45 minutes at room temperature before detecting digoxygenin by the reaction with an anti-digoxigenin alkaline phosphatase antibody (1:100) (Roche Diagnostics) in blocking solution for 60 minutes at room temperature. The inclusion of $1 \%$ Triton-x-100 at this stage helped to block any nonspecific antibody binding. This was followed by two washes 


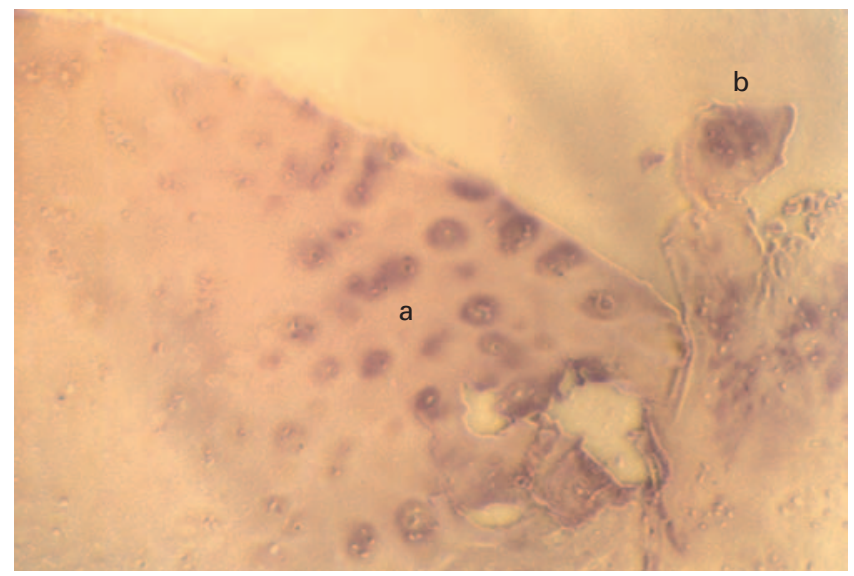

Fig. 6

Photomicrograph showing a) in situ hybridisation with positive staining for collagen type IIa around the deep zone chondrocytes and b) subchondral bone (collagen type-IIa antisense probe $\mathrm{x} 5$ ).

in maleic acid buffer with $0.3 \%$ Tween-20 and a single wash in distilled water. The hybridisation signal in the sections was detected with 5-bromo-4-chloroindolyl phosphate (BCIP)/nitro blue tetrazolium (NBT) (Sigma) which was allowed to develop in darkness overnight at $37^{\circ} \mathrm{C}$. The development was stopped by briefly rinsing the section twice with distilled water. The sections were then air dried and mounted in aqueous mountant (BDH).

They were examined by light microscopy for the presence of positive staining indicating the presence of type-IIa and type-II $b$ collagen and their location within the tissue was noted (Figs 6 to 8).

\section{Results}

The mean follow-up of the patients was $33 \pm 9$ months (SD). Biopsies were undertaken at a mean of $11 \pm 3.81$ months after the operation.

Using the Brittberg clinical rating, which relies on variables of pain, swelling and locking, three patients had excellent results, with five good, four fair and two poor. All had been rated as 'poor' pre-operatively. A simple subjective patient rating showed that 11 of the 14 knees were better at one year after operation, with two the same and one worse. Therefore, $57 \%$ of patients had a good to excellent clinical outcome at one year. The mean pre-operative Tegner and Lysholm score was $2.3 \pm 1.21$ improving to $3.69 \pm 1.38$ at one year after operation. The mean Lysholm and Gillquist score improved from $41 \pm 18.19$ before to $70 \pm 22.68$ at one year after operation. The mean verbal numerical pain score improved from $7 \pm 1.54$ before to $2.5 \pm 1.9$ at one year after the operation.

Arthroscopic examination showed that the transplanted areas were level with the surrounding surface in all cases and firm to probing. There were no cases of hypertrophy of cartilage. $^{7,8}$

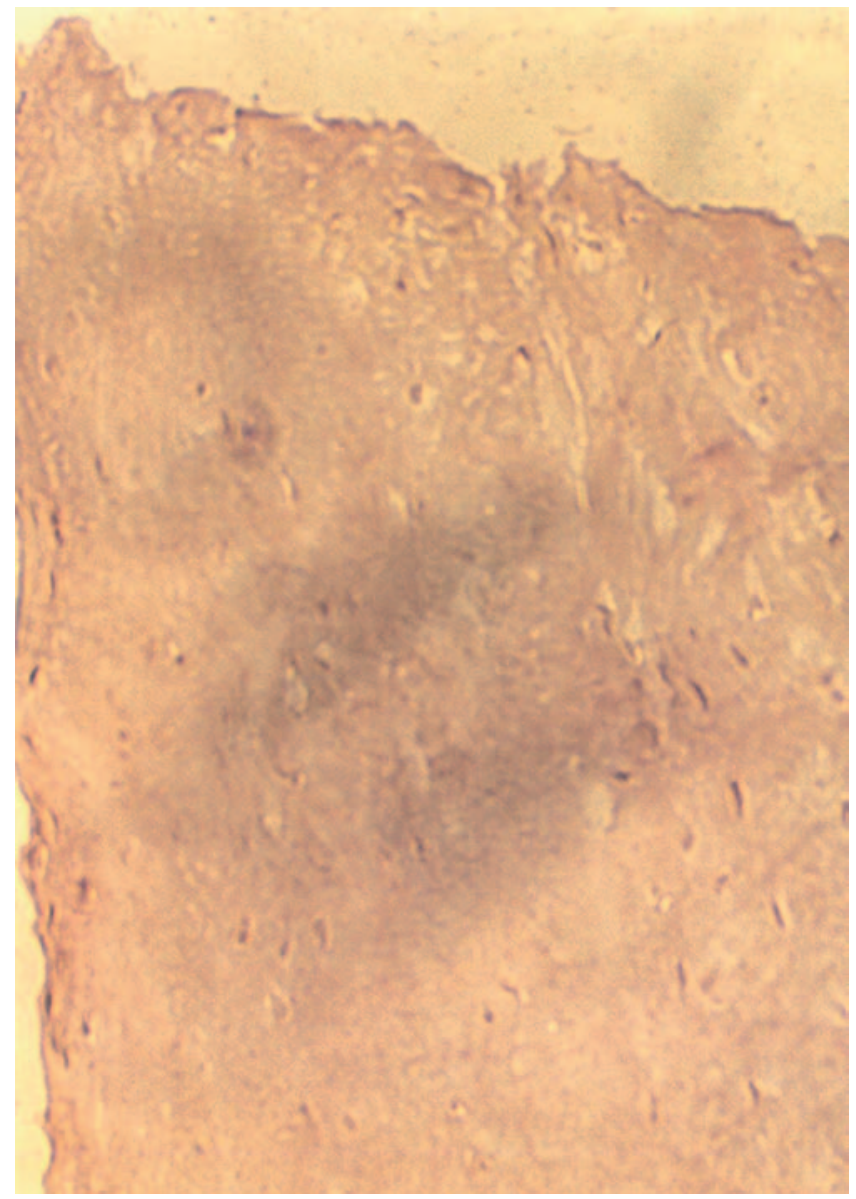

Fig. 7

Photomicrograph showing in situ hybridisation with no staining for collagen type IIa around chondrocytes in the superficial zone (collagen type-IIa antisense probe $x$ 7).

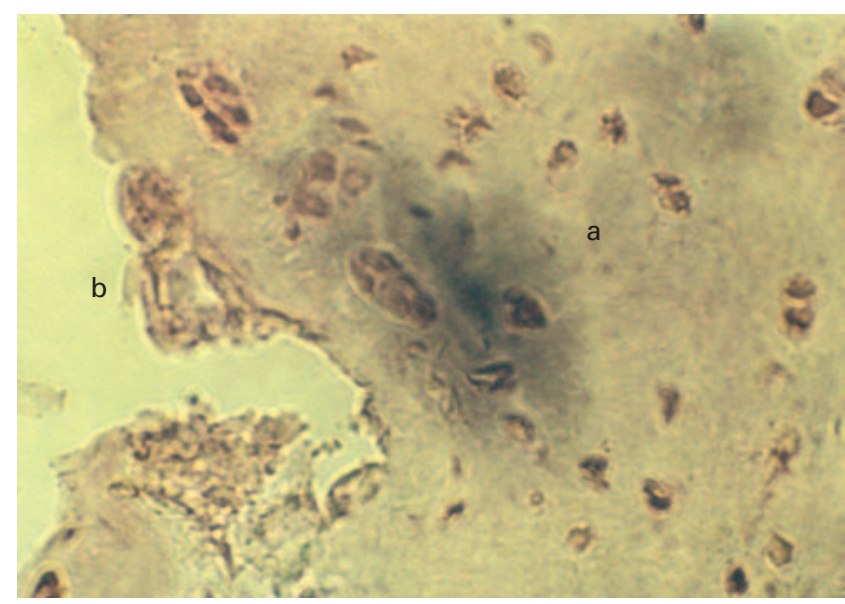

Fig. 8

Photomicrograph showing in situ hybridisation with a) positive staining for collagen type IIb round deep zone chondrocytes and b) subchondral bone (collagen type-IIb antisense probe $\mathrm{x} 7$ ). 
Table I. Correlation of the clinical data with basic hitological findings

\begin{tabular}{|c|c|c|c|c|}
\hline \multirow[b]{2}{*}{ Histology } & \multicolumn{4}{|c|}{ Brittberg rating } \\
\hline & Pre-op & Post-op & Subjective & Concurrent problems* \\
\hline Hyaline & Poor & Good & Better & OCD (stable) \\
\hline Hyaline & Poor & Good & Same & Torn LM \\
\hline Hyaline & Poor & Fair & Better & Grade I PFOA \\
\hline Hyaline & Poor & Excellent & Better & None \\
\hline Hyaline & Poor & Good & Better & None \\
\hline Hyaline with a small foci of fibrocartilage & Poor & Poor & Better & OCD (stable) \\
\hline Hyaline foci im mainly fibrous tissue & Poor & Poor & Worse & Grade III OA \\
\hline Hyaline foci and fibrocartilage & Poor & Excellent & Better & None \\
\hline Vascular fibrous tissue & Poor & Fair & Better & None \\
\hline Cellular fibrous tissue & Poor & Fair & Better & Deficient ACL \\
\hline Fibrocartilage & Poor & Fair & Better & None \\
\hline Fibrocartilage & Poor & Good & Same & None \\
\hline Fibrocartilage & Poor & Excellent & Better & None \\
\hline
\end{tabular}

For each case, histological examination of the curetted defect was performed. This showed a varying degree of degenerative changes affecting the cartilage. The base of the sample consisted of a narrow layer of subchondral bone confirming that the full thickness of degenerative cartilage had been removed.

Basic histological examination showed that six patients had hyaline cartilage only, two had foci of hyaline cartilage within fibrocartilage (mixed tissue), four fibrocartilage only and two fibrous tissue. The eight patients who showed some form of hyaline cartilage were all found on immunolocalisation to contain type- $X$ collagen in the deepest layers. TypeII collagen was localised in all eight patients with hyaline cartilage and three of those with fibrocartilage in the middle section of the biopsy sample. In situ hybridisation revealed that all the samples, regardless of histological appearance, had the potential to express both type-IIa and type-IIb collagen.

Analysing the outcome of the Brittberg rating, of the three patients who had an excellent outcome, one had hyaline cartilage, one had fibrocartilage and one had fragments of hyaline cartilage in fibrocartilage. Of the five with a good outcome, three had hyaline cartilage and two had fibrocartilage. Of the four with a fair outcome, one had hyaline cartilage, one had fibrocartilage and two had just fibrous tissue. Of the two with a poor outcome, one (hyaline cartilage) had a second stable osteochondral defect which was deemed to be unsuitable for treatment and the other (mixed tissue) had a grade-III osteoarthritic lesion on the opposite articulating surface (kissing lesion).

The only complication which occurred was a case of infection after the biopsy. The patient is now fully recovered and has gone on to have an excellent result.

\section{Discussion}

Hyaline cartilage, when damaged, has a limited ability to repair itself. It has been suggested that this is due to the ina- bility of the chondrocytes to migrate to the damaged area and to regenerate cartilage matrix. Poor blood supply may be a contributing factor. ${ }^{6}$ Using cell culture, however, it has been possible to release these cells from their matrix and replicate large numbers of autologous chondrocytes. The technique of reimplantation of these cells into a chondral defect has been shown by numerous studies to produce an improvement in the patient's symptoms. ${ }^{7,8,10}$

The terminology used in the literature to describe the cartilage regenerated by autologous chondrocyte implantation is hyaline-like. Our findings show that the regenerated tissue has all the features of hyaline cartilage. The architecture, however, lacks the organisation of articular cartilage in that the superficial zone is replaced by fibrocartilage. ${ }^{7-10}$ The results of immunolocalisation, however, show that the constituents of the collagenous matrix are distributed as in normal articular cartilage with type-II collagen in the middle of the core and type-X collagen at the base of the core near the osteochondral junction.

Our results show that in all 14 biopsies, irrespective of histological appearance, we were able to demonstrate mRNA for type-IIa and type-IIb collagen. This suggests that the cells present after autologous chondrocyte implantation are a mixture of immature chondroprogenitor phenotype and mature chondrocytes. This may represent reparative proliferation continuing one year after implantation. The clinical data in the literature support these findings and show that patients continue to improve a number of years after their initial procedure. ${ }^{7-9}$ It remains to be seen if this technique eventually results in sufficient chondrocyte organisation to allow the superficial layer to develop, thus resembling normal articular cartilage.

It has been shown that the biomechanical properties of the neocartilage are inferior to those of normal articular cartilage. ${ }^{10,22,23}$ Our findings suggest that there is potential for this cartilage to mature, given the right environmental and biomechanical factors. The length of the time which the process of maturation takes is unknown. Further studies will 
need to be carried out to assess when maturation of these cells occurs. This can be done by assessing the stage at which cells stop producing type-IIa collagen and only produce type-IIb collagen.

The sample is too small to correlate reliably the clinical outcome with the histological result but is shown in Table I.

We have also shown that it is possible to obtain neocartilage without the use of periosteum. Brittberg et al ${ }^{8}$ suggest that periosteum has a role to play in enhancing the quality of the cartilage formed. They also report cases of hypertrophy of the periosteum requiring debridement. Using the inert type-I/III collagen membrane we have so far not seen any cases of calcification or hypertrophy of the graft.

We accept that the tissue-engineered cartilage lacks the complete architectural organisation of articular cartilage, and may be presumed to have inferior biomechanical properties to normal articular cartilage at this early stage.

However, we have shown that one year after the initial implantation using an inert membrane the chondrocytes have the capability of producing a major component of articular hyaline cartilage. In addition to this, we have shown that chondrocytes are of an immature phenotype and presumably may continue to proliferate and mature one year after initial implantation. We have also demonstrated by immunolocalisation of type-II and type-X collagen, that the distribution of these components closely resembles that of normal articular hyaline cartilage.

We would like to acknowledge the help and support of Professor Ming H. Zheng, Director of Research and Professor David Wood, Head of Unit, at the University of Western Australia.

No benefits in any form have been received or will be received from a commercial party related directly or indirectly to the subject of this article.

\section{References}

1. Johnson LL. Arthroscopic abrasion arthroplasty: a review. Clin Orthop 2001;(Suppl 391):306-17.

2. Pridie KW. A method of resurfacing osteoarthritic knee joints. J Bone Joint Surg [Br] 1959;41-B:618-9.

3. Muckle DS, Minns RJ. Biological response to woven carbon fibre pads in the knee: a clinical and experimental study. J Bone Joint Surg $[\mathrm{Br}]$ 1990;72-B:60-2.

4. Meister K, Cobb A, Bentley G. Treatment of painful articular cartilage defects of the patella by carbon fibre implants. J Bone Joint Surg [Br] 1998;80-B:965-70.
5. Steadman RJ, Rodkey WG, Rodrigo JJ. Microfracture: surgical technique and rehabilitation to treat chondral defects. Clin Orthop 2001;(Suppl 391):362-9.

6. Akeson WH, Bugbee W, Chu C, Giurea A. Differences in mesenchymal tissue repair. Clin Orthop 2001;(Suppl 391):124-41.

7. Peterson L, Minas T, Brittberg M, et al. Two- to 9-year outcome after autologous chondrocyte transplantation of the knee. Clin Orthop 2000;374:212-34.

8. Brittberg M, Lindahl A, Nilsson A, et al. Treatment of deep cartilage defects in the knee with autologous chondrocyte transplantation. N Engl J Med 1994;331:889-95.

9. Grande DA, Pitman MI, Peterson L, Menche D, Klein M. The repair of experimentally produced defects in rabbit articular cartilage by autologous chondrocyte transplantation. J Orthop Res 1989;7:208-18.

10. Peterson L, Brittberg M, Kiviranta I, Akerlund EL, Lindahl A. Autologous chondrocyte transplantation: biomechanics and long-term durability. Am J Sports Med 2002;30:2-12.

11. Richardson JB, Caterson B, Evans EH, Ashton BA, Roberts S. Repair of human articular cartilage after implantation of autologous chondrocytes. J Bone Joint Surg [Br] 1999;81-B:1064-8.

12. Roberts S, Hollander AP, Caterson B, Menage J, Richardson JB. Matrix turnover in human cartilage repair tissue in autologous chondrocyte implantation. Arthritis Rheum 2001;44:2586-98.

13. Poole AR, Kojima T, Yasuda T, et al. Composition and structure of articular cartilage: a template for tissue repair. Clin Orthop 2001; (Suppl 391):S26-33.

14. Buckwalter JA, Mankin HJ. Articular cartilage: tissue design and chondrocyte-matrix interactions. Instr Course Lect 1998;47:477-86.

15. Bruckner P, van der Rest M. Structure and function of cartilage collagens. Microsc Res Tech 1994;28:378-84.

16. Aigner T, Zhu Y, Chansky HH, et al. Re-expression of type IIa procollagen by adult articular chondrocytes in osteoarthritic cartilage. Arthritis Rheum 1999;42:1443-50.

17. Ehlers EM, Fuss M, Rohwedel J, et al. Development of a biocomposite to fill out articular cartilage lesions: light, scanning and transmission electron microscopy of sheep chondrocytes cultured on a collagen I/III sponge. Anat Anz 1999;181:513-8.

18. Tegner Y, Lysholm J. Rating systems in the evaluation of knee ligament injuries. Clin Orthop 1985;198:43-9.

19. Lysholm J, Gillquist J. Evaluation of knee ligament surgery results with special emphasis on the use of a scoring scale. Am J Sports Med 1982;10:150-3.

20. Chambers MG, Kuffner T, Cowan SK, Cheah KS, Mason RM. Expression of collagen and aggrecan genes in normal and osteoarthritic murine knee joints. Osteoarthritis Cartilage 2002;10:51-61.

21. Flannelly J, Chambers MG, Dudhia J, et al. Metalloproteinase and tissue inhibitor of expression in the murine STR/ort murine model of osteoarthritis. Osteoarthritis Cartilage 2002;10:722-33.

22. Duda GN, Haisch A, Endres M, et al. Mechanical quality of tissue engineered cartilage: results after 6 and 12 weeks in vivo. J Biomed Mater Res 2000;53:673-7.

23. Hasler EM, Herzog W, Wu JZ, Muller W, Wyss U. Articular cartilage biomechanics: theoretical models, material properties, and biosynthetic response. Crit Rev Biomed Eng 1999;27:415-88. 